\title{
A QoS framework for Next Generation Networks Based on Metro Ethernet
}

\author{
Elyas Mohamadzadeh Kosari, Mohammad Hossein Yaghmaee Moghaddam, Maryam Taheri Tehranian, \\ and Edris Shahany
}

\begin{abstract}
Nowadays, the interest in using Metro Ethernet as the main access technology in Next Generation Networks (NGN) is ever increasing. One of the main demands of these networks is to provide Quality of Service $(\mathrm{QoS})$ for multimedia and other time stringent applications. At present, several well-known technologies are used by the metro Ethernet, such as NG-SONET/SDH, VPLS, and RPR, which have been used independently so far, and none of them provides QoS. ETSI TISPAN architecture is used to provide QoS in NGN. In this paper we have used three different metro Ethernet technologies, NG-SONET/SDH, RPR, and VPLS in a combination with RACS and NASS (ETSI TISPAN subsystems) to meet QoS requirement. To do so, we propose a framework which uses the mentioned technologies and TISPAN to satisfy QoS in metro Ethernet networks.
\end{abstract}

Index Terms-Metro ethernet, next generation network, quality of service, TISPAN.

\section{INTRODUCTION}

The concept of Next Generation Network (NGN) provides a new network infrastructure with features and capabilities that support the provision of value-added multimedia services over multiple and heterogeneous Quality of Service (QoS) enabled transport technologies.

The idea of a NGN is commonly understood as a new kind of network architecture together with its related technologies. An NGN architecture is developed with the purpose of integrating different multiple services (data, voice, video), called Triple-Play services, and of facilitating the convergence of fixed and mobile networks.

There are different access technologies such as Metro Ethernet, wireless, WiMAX, Fiber Distributed Data Interface (FDDI), in NGN which are connected to an IP core network. These access technologies require QoS to provide Triple-Play services. Therefore, QoS provisioning is one of the most important challenges of NGN.

ETSI, one of NGN standardization institutes, presents Telecommunications and Internet converged Services and

Manuscript received January 22, 2012; revised March 4, 2012.

This research has been sponsored by Iran Telecom Research Center (ITRC) under contract NO. T/500/9966.

E. M. Kosari and M. H. Y. Moghaddam are with the Department of Computer, Ferdowsi University of Mashhad, Mashhad, Iran (e-mail: elyas.kosari@stu-mail.um.ac.ir, yaghmaee@ieee.org).

M. T. Tehranian is with Department of Computer and Information Technology, Mazandaran University of Science and Technology, Babol, Iran (e-mail: mtaheri@ustmb.ac.ir).

E. Shahany is with Department of Electerical, Computer and Information Technology, Islamic Azad Univesity of Qazvin, Qazvin, Iran (e-mail: e.shahani@qiau.ac.ir).
Protocols for Advanced Networking (TISPAN) architecture [1] to provide QoS in NGN. TISPAN subsystems are Resource and Admission Control Subsystem (RACS) [2] and Network Attachment SubSystem (NASS) [3], which play important roles in providing QoS and security to NGN, respectively.

Metro Ethernet is main access network in NGN. Metropolitan networks play a critical role in the overall expansion of network services. They not only provide for services within individual metropolitan areas, but they also serve as the gateways for wide-area national- and international-scale networks.

Since metro Ethernet is the main access technology in NGN, it is necessary to provide QoS for these kinds of networks. At present, there exist several well-known technologies used by the metro Ethernet, such as Next Generation SONET/SDH (NG-SONET/SDH) [4-6], Virtual Private LAN Service (VPLS) [7, 8], and Resilient Packet Ring (RPR) [9], which being used independently so far.

In this paper, we combine these technologies to propose a QoS framework based on TISPAN architecture. The layout of this paper is as follows. Section 2 describes the proposed QoS framework, based on metro Ethernet technologies and TISPAN subsystems, RACS and NASS. Section 3 describes the most important conclusions achieved along the paper.

\section{The Proposed QoS Framework}

Today different kinds of technologies are being used in metro Ethernet networks such as NG-SONET/SDH, RPR, and VPLS. The combination of these technologies could bring some advantages to the metro Ethernet networks such as the utilization of NG-SONET/SDH as the future backbone of telecommunications industry, and the possibility of providing effective communication with other technologies. Despite these advantages, providing QoS is still one of the main challenges of these networks. Therefore, ETSI TISPAN architecture can be used as a control plane to provide Quality of Service (QoS) to the network.

NG-SONET/SDH technology is used in Physical Layer (L1), RPR technology is used in Medium Access Control (MAC) sublayer in Data-Link Layer (L2), and VPLS is used in L2 of the OSI reference model, that each one are used independently. Therefore, we propose a stack protocol as a framework for metro Ethernet, which utilizes these technologies simultaneously. Since RACS and NASS use IP addresses, we put them in Network Layer (L3) of the proposed framework. Fig. 1 illustrates the proposed stack protocol. 
The proposed protocol stack, Fig. 1, works as follows: The application specifies its QoS requirements and sends a request to control plane. The operations in the control plane are done in two parts. NASS checks the request for Authentication, Authorization, and Accounting (AAA), and RACS checks if the available resources could meet the requested requirements. If the request is accepted, RACS reserves the resources and sends an Acknowledgement (ACK) to the application regarding to this action so that the application could send its packets to lower layers to be transported to the network.

In the following subsections, we describe each layer's operation in more details.

\section{A. NG-SONET/SDH}

NG-SONET/SDH plays an important role in metro networks. SONET/SDH technologies constitute the core transport infrastructure of major telecom service providers worldwide and, since various services such as Ethernet, IP, and voice must be composed in the core network for transport, equipments of such networks must have the ability of adaption of interfaces of all used technologies in the metro arena. These equipments are known as Optical Edge Devices (OED) and/or Multi-Service Provisioning Platform (MSPP).

NG-SONET/SDH backbone uses ring topology in order to provide more flexibility and protection in large networks and it uses OC-48 lines with data rates at $2.5 \mathrm{Gbps}$ to provide high speed connections.

NG-SONET/SDH extends the utility of the existing SONET/SDH network by leveraging existing layer 1 networking and including technologies such as virtual concatenation (VCAT), generic framing procedure (GFP), and the link capacity adjustment scheme (LCAS) (See Fig. 2).

In NG-SONET/SDH backbone an MSPP is a multi-service node that can fulfill the access, process, and transport of TDM, ATM, and Ethernet services together, and provides the unified network management platform for all of these services.

GFP is the most popular encapsulation protocol today to adapt a variety of character and packet oriented payloads into SONET/SDH containers. Low and deterministic overhead, robust delineation mechanism and extensibility to support client-specific management functions are the main characteristics of this protocol.

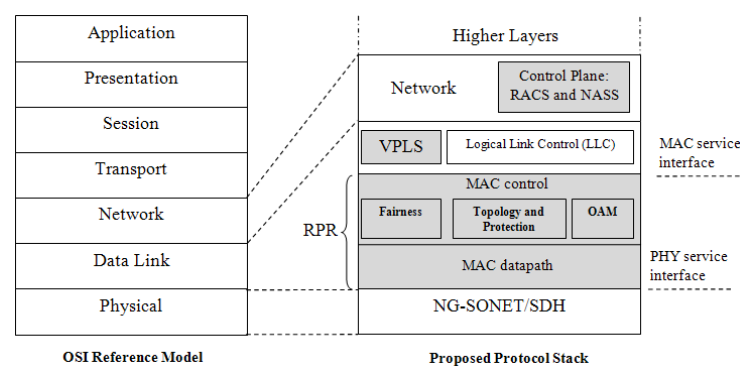

Fig. 1. Proposed protocol stack relationship to the OSI reference model.

VCAT has been expanded with a protocol called LCAS that enhances the VCAT scheme with hitless in-service addition and removal of STSs/VCATs to/from the Virtual Concatenation Group (VCG). Additionally, the LCAS protocol provides load sharing protection by dynamically removing failed members from the VCG if and when they experience faults. This important LCAS functionality allows a provider to significantly improve the resiliency offered to end users by provisioning diversely routed SONET/SDH paths that belong to the same VCG.

NG-SONET/SDH's flexibility enables network operators to either build a network using hybrid TDM/packet multiservice provisioning platforms or to provide only the underlying transmit bit stream framing.

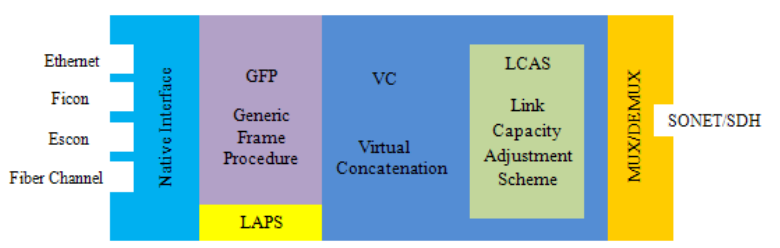

Fig. 2. An overview of NG-SONET/SDH.

With the telecommunications' industry in transition, the emphasis within network operations is on meeting customer demand within strict budgets. NG-SONET/SDH not only is cost-effective, it enhances legacy networks' capabilities and capacity to levels that surpass other options. By integrating NG-SONET/SDH technology into legacy networks, significant gain in throughput, quality, and service availability are achieved.

\section{B. $R P R$}

RPR is a MAC layer for metro-ring networks, devised to achieve objectives such as high throughput, fault tolerance, and bandwidth efficiency, which are not simultaneously achieved in current technologies [10].

It is a high-speed MAC technology for metropolitan ring networks, which supports three Class of Services (CoS): Class A (Real-Time), Class B (near Real-Time) and Class C (Best Effort). RPR has dual counter-rotating rings connecting $\mathrm{N}$ nodes, ringlet 0 clockwise and ringlet 1 counterclockwise, as shown in Fig 3 [9].

Each node connects to both ringlets and has station ingress and egress traffic. Ringlet ingress traffic is checked to determine if it is destined locally to the egress traffic, or pass-through where it is queued in the transit buffer (TB). The RPR standard [9], defines two implementations: single queue (or 1-TB) called Primary Transit Queue (PTQ) serviced with strict priority over ingress buffer traffic. The second is with dual queues (or 2-TB), PTQ for Class A traffic, and Secondary Transit Queue (STQ) for Class B and Class C traffic. Traffic in STQ is service with strict priority over ingress buffer traffic when a certain depth (or threshold) is reached in the STQ. Otherwise, STQ traffic is serviced using a round-robin discipline with ingress buffer traffic [11].

The RPR MAC is based on Buffer Insertion Ring (BIR) [12][13] which operates as follows. There is an insertion buffer at every node interface to solve the conflict between the data already flowing on the ring and the data ready to be transmitted by a node. Ring traffic has non-preemptive priority over node traffic. If upstream nodes keep sending traffic, downstream nodes may experience so-called starvation problem since upstream traffic prevent downstream nodes from accessing the ring. To avoid 
starvation problem, a fairness mechanism is required to regulate ring access.

The fairness algorithm uses explicit rate feedback to control the amount of traffic that each node inserts on the ring. There are two modes in RPR: Aggressive Mode (AM) and Conservative Mode (CM). The AM is the default mode and is associated with the 2-TB design, while the CM uses the 1-TB implementation. In both modes, each node measures the output of the scheduler and ingress traffic over a fixed interval. These measurements are used to detect congestion and compute fair rates to be sent in control messages to upstream nodes [14].

RPR provides QoS with packet prioritization and offers higher throughput as it allows spatial reuse. The spatial reuse is achieved through destination-removal. Unlike the source-removal employed in token ring, destination-removal allows the destination node to remove the packet from the ring, and thus, enables concurrent transmissions over different segments of a ring. As a result, the total ring throughput of a spatial reuse packet ring can be significantly higher than the capacity of a single link. However, since the ring bandwidth becomes a shared medium, a key challenge is to design a MAC scheme that ensures all nodes have fair access to the ring [14], [15].

As illustrated in the proposed framework in Fig 1, RPR sublayer is divided into two sublayers itself: MAC control and MAC datapath.

The MAC control sublayer supports control activities necessary to maintain the state of the MAC and datapath activities not identified with a particular ringlet. The control activities are distributed among stations on the ring in order to survive any single point of failure. Control entities in a station communicate with peer control entities in other stations using the services of the MAC datapath sublayer [9].

The MAC datapath sublayer provides the interactions between the client and the physical layer and the communication between peer datapath sublayers in other MACs on the ring. It includes a single ringlet selection entity and two distinct instances of ringlet-specific datapaths. The ringlet selection entity determines the ringlet to be used to transmit the frame. The ringlet-specific instance provides encapsulation and decapsulation of client data frames on transmit and receive, copying and routing received frames to the Logical Link Control (LLC) and MAC control sublayers, and deleting frames from the ring on error or time-out [9].

In the following paragraphs, the MAC interfaces [9] to LLC and NG-SONET/SDH layer in the proposed framework are described.

The MAC service interface provides service primitives used by MAC clients to exchange data with one or more peer clients, or to transfer local control information between the MAC and the LLC.

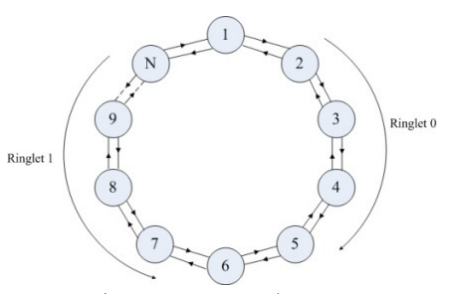

Fig. 3. RPR topology
The MAC physical layer service interface allows the MAC to transfer information to and from the NG-SONET/SDH layer and physical layer interfaces (PHYs) through logical service primitives.

\section{VPLS}

Metro Ethernet services are often point-to-point connections between multiple sites within the same metro. However, the ultimate vision held by Metro Ethernet proponents is the ability to move beyond point-to-point connectivity that is confined to a single metro area to deliver point-to-multipoint or multipoint-to-multipoint connectivity either within a single metro or spanning multiple metro areas. In other words, make all sites appear if they are connected to the same simple Ethernet LAN, irrespective of whether the sites are in the same metro area or spread across multiple metro areas. This is known as VPLS, which provides both intra- and inter-metro Ethernet connectivity over a scalable IP/MPLS service provider network. VPLS is a multipoint Layer 2 Virtual Private Network (VPN) technology that allows multiple remote sites to be connected over an emulated Ethernet broadcast domain across an IP/MPLS provider network.

VPLS provides Ethernet connectivity between any customer site to any customer site, some or all customer sites. Therefore, from the customer perspective the service provider network looks like a virtual Ethernet switch connecting the customer's remote sites [16].

In a VPLS scenario the customer device, referred to as Customer Edge (CE), is connected through a single Ethernet connection (typically a VLAN) to the PE router that forwards the customer frames to the appropriate remote PE serving the destination customer site. Transport tunnels, typically implemented as MPLS-based Label Switched Paths (LSPs), are required between the PEs to transfer the traffic flows generated by the VPLS customers [17]. Fig. 4 shows the VPLS reference model.

There are two VPLS implementations supported by the IETF, [7] uses BGP signaling and auto-discovery, while [8] uses LDP signaling. Since BGP-based implementation provides the highest level of automation and operational efficiency, it's been used in the proposed framework.

Auto-Discovery, in BGP-based implementation, allows PE routers to find each other in a VPLS instance (that is, a VPLS for a particular enterprise). According to [7], when a new PE joins to the VPLS, a BGP-session is established between the new PE and the Route Reflector (RR), and one or more ports are associated to that PE. Then, the new PE advertises that it's part of the VPLS instance via RR to other PEs that are joined to that VPLS domain.

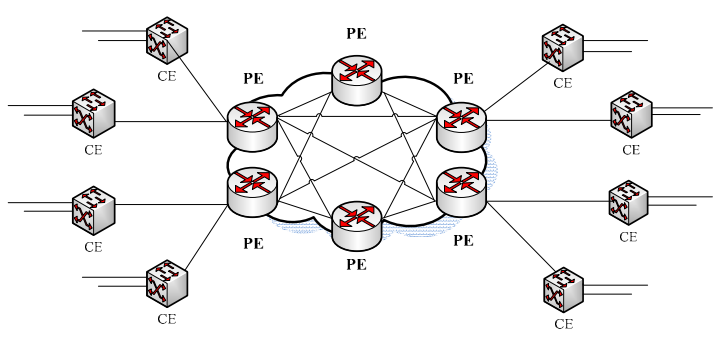

Fig. 4. VPLS reference model. 
The proposed framework defines VPLS communication with RPR based on RPR's shared media and native multicasting capabilities to emulate a common broadcast domain for all subscribers in a VPLS instance. VPLS over RPR implementation [18] is unique in that it offers the benefits derived from combining MPLS and RPR, such as efficient statistical multiplexing, spatial reuse, sub-50msec protection switching, control plane provisioning and MPLS traffic engineering.

VPLS packets are sent as MPLS Pseudo-Wire (PW) packets, relying on the MPLS PW label to define the VPLS ID of the service instance. A pseudo-wire is an emulation of a layer 2 point-to-point connection-oriented service over a Packet-Switching Network (PSN).These are transported over the RPR shared media using an RPR multicast address, resulting in a single packet being sent to the ring, where every transit node will examine the RPR multicast packet before forwarding a copy back to the ring. Upon examining the RPR multicast packet, the transit node verifies whether the VPLS ID is locally configured, in which case a copy of the packet is sent to the local node prior to forwarding it back into the RPR ring.

\section{RACS and NASS}

In addition to IP/MPLS in Layer 3 of the proposed framework, we need a control sublayer to provide Quality of Service (QoS) to the network and the usage of RACS and NASS is a good selection.

RACS and NASS are the two important subsystems of ETSI TISPAN architecture and have a significant role in QoS control in this architecture.

RACS in this technology, which plays the role of control sublayer for the Metro Ethernet Networks, presents the following functionalities for each resource reservation session:

- Policy Control (PC): Service-based Policy Decision Function (SPDF) in the core network and A-RACF in the access network use a set of rules and policies to determine the way the requests are satisfied.

- Admission Control (AC): Access - Resource Admission Control Function (A-RACF) according to available resources checks if the QoS requirements of the access network are satisfied. In fact, RACS determines whether a request could be accepted and appropriate transport resources could be assigned to it, based on the information related to accessibility of the resources and other policy rules such as priorities.

- Resource Reservation: RACS implements a resource reservation mechanism which allows the applications to request carrier resources from access, aggregate and core networks. A-RACF in the access network reserves the resources for the request.

- NAT/Gate Control: SPDF in the edge of core networks and A-RACF in the edge between access and core networks, do gate control functions and control the NAT functionalities.

NASS functionalities and functional entities are:

- Providing IP addresses and other dynamic configuration parameters of the terminal which are done by Network Access Configuration Function (NACF).
- Identifying and authentication of the user in IP layer before or during IP assignment procedure by User Access Authorization Function (UAAF).

- Authorizing the user to access the network according to his/her profile which is done by the UAAF based on the information in Profile Data Base Function (PDBF) databases.

To provide the above services, RACS and NASS need to communicate with different network layers and such communication in achieved by using different protocols such as DIAMETER [19], RADIUS, Dynamic Host Configuration Protocol (DHCP), etc.

DHCP protocol, which is used to assign IP addresses and to configure other network parameters, provides the connection between NASS components and the network. Moreover, NASS uses RADIUS server for authentication.

RACS does admission control and resource reservation operations, by using DIAMETER commands, and thus plays the role of control sublayer in the proposed framework. The main commands of DIAMETER protocol are summarized in Table I [19], and QoS commands in DIAMETER are summarized in table II [20].

\section{CONCLUSION}

There has been a significant growth in the application of Metro Ethernet Networks in NGN, thus QoS provisioning in these networks is crucial. Since the ETSI TISPAN architecture is used to provide QoS in NGN, it could be used in metro Ethernet networks.

TABLE I: MAIN COMMANDS OF DIAMETER PROTOCOL
\begin{tabular}{|l|l|l|}
\hline \multicolumn{1}{|c|}{ Command Name } & Abbreviation & Code \\
\hline Re-Auth-Request & RAR & 258 \\
\hline Re-Auth-Answer & RAA & 258 \\
\hline Abort-Session-Request & ASR & 274 \\
\hline Abort-Session-Answer & ASA & 274 \\
\hline Session-Term-Request & STR & 275 \\
\hline Session-Term-Answer & STA & 275 \\
\hline
\end{tabular}

TABLE II: QOS COMMANDS IN DIAMETER

\begin{tabular}{|l|l|c|}
\hline \multicolumn{1}{|c|}{ Command Name } & \multicolumn{1}{c|}{ Abbreviation } & Code \\
\hline QoS-Authorization-Request & QAR & {$[$ TBD1] } \\
\hline QoS- Authorization-Answer & QAA & {$[$ TBD2] } \\
\hline QoS-Install-Request & QIR & {$[$ TBD3] } \\
\hline QoS- Install- Answer & QIA & {$[$ TBD4] } \\
\hline
\end{tabular}

In this paper we proposed a combined framework of the three technologies, NG-SONET/SDH, RPR, and VPLS, for metro Ethernet networks, which has some advantages for NGN such as high data transport rate (provided by NG-SONET/SDH), high throughput, fault tolerance, bandwidth efficiency, and fairness (provided by RPR), highest level of automation and operational efficiency (provided by VPLS). However, slow convergence of the fairness algorithm is still a challenge in RPR, and there are some arguments against BGP-base VPLS due to its complexity and the need for pre-block allocation of labels.

In addition, RACS and NASS are used as a control plane to provide QoS in the proposed framework. These components by utilizing protocols including DIAMETER, RADIUS, and DHCP, perform operations such as AAA, call admission 
control, resource reservation, and IP allocation to provide QoS and security to the network.

\section{ACKNOWLEDGMENT}

The research team wishes to express their gratitude to Iran Telecom Research Center for their valuable help and support.

\section{REFERENCES}

[1] ETSI ES 282001 V3.4.1, "Telecommunications and Internet converged Services and Protocols for Advanced Networking (TISPAN); NGN Functional Architecture," September 2009

[2] ETSI ES 282003 V3.4.2, TISPAN; Resource and Admission Control Subsystem (RACS); Functional Architecture,” April 2010.

[3] ETSI ES 282004 V3.4.1, TISPAN; Network Attachment Subsystem (NASS); Functional Architecture," March 2010.

[4] ITU-T Recommendation G.7041, Generic Framing Procedure (GFP), 2006

[5] ITU-T Recommendation G.707, Virtual Concatenation (VCAT for SONET/SDH), 2006

[6] ITU-T Recommendation G.7042, Link Capacity Adjustment Scheme (LCAS), 2006

[7] IETF RFC 4761, "Virtual Private LAN Service (VPLS) Using BGP for Auto-Discovery and Signaling".

[8] IETF RFC 4762, "Virtual Private LAN Service (VPLS) Using Label Distribution Protocol (LDP) Signaling"

[9] IEEE Std. 802.17, Resilient packet ring (RPR) access method and physical layer specification

[10] F. Davik, M. Yelmaz, S. Gjessing, and N.Uzun, "IEEE 802.17 Resilient packet rings tutorial" IEEE Communications Magazine, March 2004.

[11] H. Tang, I. Lambadaris, H. Mehrvar, J. Talim, and A. Matrawy, "A new access control scheme for metropolitan packet ring networks," IEEE Communications Society, Globecom 2004, pp. 281-287, 2004.

[12] W. Bux and M. Schlater, An approximation method for the performance analysis of Buffer Insertion Rings, IEEE Trans. on Communications, vol. 31, no. 1, pp. 50-55, January, 1983.

[13] E. Hafner, Z. Nenadal, and M. Tschanz, "A Digital Loop Communication Systems," IEEE Trans. on Communications, COM-22, 6, pp. 877-881, June 1974.

[14] V. Gambiroza, P. Yuan, L. Balzano, Y. Liu, S. Sheafor, and E. Knightly, "Design, analysis, and implementation of DVSR: a fair high-performance protocol for packet rings," IEEEIACMTrans, on Networking, vol. 12, no. 1, pp. 85-102, February, 2004

[15] F. Davik et al "IEEE 802.17 Resilient Packet Ring Tutorial," IEEE Comm. Magzine, March 2004.

[16] N. AbuAli, H. T. Mouftah, and S. Gazor, "Multi-Path Traffic Engineering Distributed VPLS Routing Algorithm," Department of Electrical and computer Engineering Queen's University 2005

[17] B. Martini, V. Martini, F. Baroncelli, P. Castoldi, L. Rea, A. Valenti, and F. Matera, "Dynamic QoS Control Based on VPLS in Service Oriented Transport Networks" 2008

[18] L. Aguirre-Torres and G. Rosenfeld, "High-Availability in Multipoint to Multipoint Ethernet for the Delivery of Triple Play Services," Optical Fiber Communication Conference, OFC 2006
[19] IETF RFC 3588: "Diameter Base Protocol "

[20] D. Sun, EdAlcatel-Lucent, P. McCann Motorola Labs, H. Tschofenig Nokia Siemens Networks T. Tsou Huawei, A. Doria Lulea University of Technology G. Zorn, and Ed. Network Zen "Diameter Quality of Service Application draft-ietf-dime-diameter-qos-15” March 7, 2010

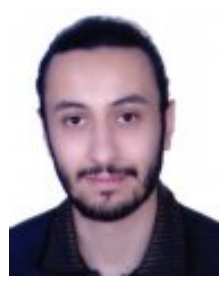

Elyas Mohamadzadeh Kosari was born in 1985, Mashhad, Iran. He received his bachelor degree in computer engineering-software in Islamic Azad University of Shirvan, Shirvan, Iran. He is currently pursuing his M.S. degree in computer engineering in Ferdowsi University of Mashhad, Mashhad, Iran.

Elyas has worked in many computer science fields including Programming. His research interests include quality of service and congestion control. $\mathrm{He}$ is currently working on congestion control in RPR networks.

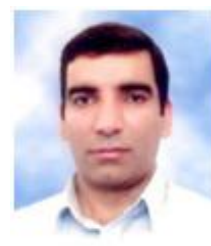

Mohammad Hossien Yaghmaee was born in July 1971 in Mashhad, Iran. He received his B.S. degree in Communication Engineering from Sharif University of Technology, Tehran, Iran in 1993, and M.S. degree in communication engineering from Tehran Polytechnic (Amirkabir) University of Technology in 1995. He received his Ph.D degree in communication engineering from Tehran Polytechnic (Amirkabir) University of Technology in 2000

Mohammad Hossein has been a computer network engineer with several networking projects in Iran Telecommunication Research Center (ITRC) since 1992. November 1998 to July1999, he was with Network Technology Group (NTG), C\&C Media research labs., NEC corporation, Tokyo, Japan, as visiting research scholar. September 2007 to August 2008, he was with the Lane Department of Computer Science and Electrical Engineering, West Virginia University, Morgantown, USA as the visiting associate professor. He is author of 3 books all in Farsi language. He has published more than 60 international conference and journal papers. His research interests are in Wireless Sensor Networks (WSNs), traffic and congestion control, high speed networks including ATM and MPLS, Quality of Services (QoS) and fuzzy logic control.

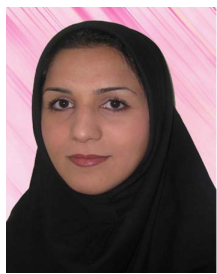

Maryam Taheri Tehranian was born in 1985 in Mashhad, Iran. She received her B.S in computer engineering-hardware in Ferdowsi University of Mashhad, Mashhad, Iran. She is now pursuing her M.S in Information Technology in Mazandaran University of Science \& Technology, Babol, Iran

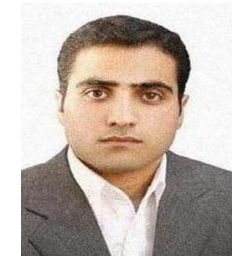

Edris Shahani was born in 1983 in Mashhad, Iran. $\mathrm{He}$ received his bachelor degree in computer engineering-hardware in Khayyam Institute of Higher Educations, Mashad, Iran. He also received his M.S degree in computer engineering- hardware in Islamic Azad Univesity of Qazvin, Qazvin, Iran 